\title{
Letaler Verlauf einer Influenza-Pneumonie und Literaturrecherche in Zeiten der wachsenden Aufmerksamkeit für das neue Coronavirus Sars-CoV-2
}

\section{Einleitung}

In Zeiten steigender Aufmerksamkeit für das neue Coronavirus Sars-CoV-2, das erstmals im zentralchinesischen Wuhan Ende 2019 nachgewiesen wurde und eine Lungenerkrankung mit dem Namen COVID-19 auslöst, sollten andere schwere Atemwegserkrankungen wie Pneumonien durch saisonale Influenzaviren oder Bakterien nicht aus dem Fokus fallen. Laut statistischem Bundesamt gingen im Jahr 2017 über $9 \%$ der Todesursachen auf eine Pneumonie zurück [Statistisches Bundesamt, Stand März 2020]. Ein häufiger Erreger ist das Influenzavirus. Der Gesundheitsbericht des Robert-Koch-Instituts gibt das saisonale Maximum der Influenza-Welle für die Monate Januar und Februar an; zur gleichen Zeit traten die ersten COVID-19-Fälle in Deutschland auf. Die Mortalität der saisonalen Influenza schwankt je nach Ausmaß der Grippewelle erheblich. Das RKI schätzt die mit Influenza in Verbindung gebrachten Todesfälle der Saison 2015/16 auf 237 und für die Saison 2017/18 auf 25 100. Eine Beurteilung der Mortalitätsrate durch Sars-CoV-2 ist zum Zeitpunkt des Verfassens dieses Artikels noch nicht abschließend möglich (Stand März 2020). Von der saisonalen Grippe besonders betroffen sind Kinder und Jugendliche, Menschen über 60 Jahre und Patienten mit chronischen Erkrankungen. Neben der klinischen und der laborchemischen Untersuchung kommt der radiografischen Diagnostik eine wichtige Rolle zu [Bericht zur Epidemiologie der Influenza in Deutschland Saison 2018/19, RKI]. Gleiches gilt für COVID-19. Damit ergibt sich eine Überschneidung der Risikogruppen, die eine besondere Aufmerksamkeit der zuständigen Ärzte im Rahmen der Erstdiagnostik erfordert.

\section{Fallbeschreibung}

Wir berichten über einen 45-jährigen Patienten, der sich Ende Januar in unserer zentralen Notaufnahme in reduziertem Allgemeinzustand, mit Fieber bis $40,5^{\circ} \mathrm{C}$, Husten, Auswurf und Dyspnoe vorstellte.
Auskultatorisch zeigten sich feinblasige basale Rasselgeräusche über beiden Pulmones; laborchemisch bestand eine CRPErhöhung auf $42,5 \mathrm{mg} / \mathrm{dl}$. Ein InfluenzaSchnelltest war negativ. Vorerkrankungen bestünden nicht. Die initiale Thorax-Röntgenübersichtsaufnahme war unauffällig ( $\triangleright$ Abb. 1). Der Patient wurde bei klinischem Verdacht einer Pneumonie anhand des CRB-65-Scores bewertet und mit oraler Antibiose sowie der Empfehlung nach Haus entlassen, in 3 Tagen einen Allgemeinmediziner zur Verlaufskontrolle zu kontaktieren. Bei ausbleibender Besserung solle vorzeitiger ein Arzt aufgesucht werden.

4 Tage später stellte sich der Patient erneut in der zentralen Notaufnahme vor. Zu diesem Zeitpunkt hatte sich die klinische Symptomatik deutlich verschlechtert, er zeigte Zeichen einer Hypoxämie. Ein erneuter Schnelltest und eine PCR waren nun positiv für Influenza A. Aufgrund des sich entwickelnden ARDS wurde der Patient intensivmedizinisch betreut, endotracheal intubiert und mit Lagerungstherapie begonnen. Das bei stationärer Aufnahme angefertigte Röntgenbild des Thorax zeigte bipulmonale fleckig-konfluierende Verschattungen; das angefertigte Thorax-CT ergab ausgedehnte, peripher und unterlappenbetonte Milchglastrübungen sowie Konsolidierungen - vereinbar mit einer Influenza-A-Pneumonie ( $\mathbf{A b b}$. 2).

Trotz forcierter intensivmedizinischer Maßnahmen verschlechterte sich der Zustand des Patienten, sodass die Anlage einer veno-venösen ECMO notwendig wurde. Im Rahmen des Schockgeschehens entwickelte der Patient ein akutes Nierenversagen. In der erneuten CT des Thorax zeigten sich zunehmende Konsolidierungen ( $\triangleright$ Abb.3). Aufgrund einer CT-morphologisch vermuteten ausgedehnten Darmischämie mit Pneumatosis intestinalis erfolgte die explorative Laparotomie, in der bei global avitalem Darm eine infauste Prognose des Patienten anzunehmen war ( $\downarrow$ Abb. 4). Kurz darauf verstarb der Patient an einem Multiorganversagen.

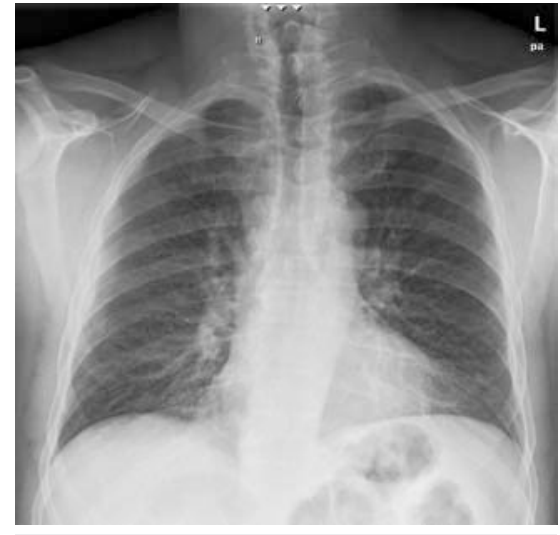

- Abb. 1 Unauffälliges initiales Röntgenbild des Thorax ohne Nachweis Pneumonie-typischer Verdichtungen.

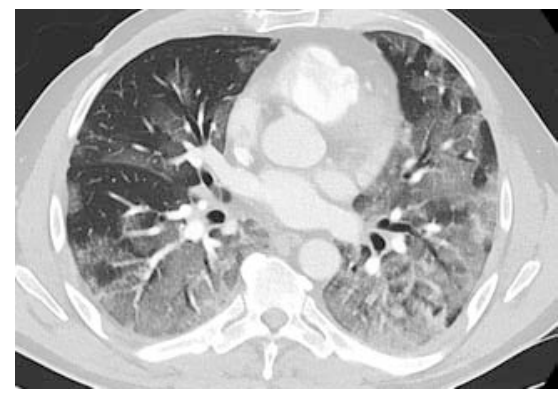

- Abb. 2 Bei Wiederaufnahme 4 Tage später zeigten sich ausgedehnte, in der Lungenperipherie und in den Unterfeldern betonte Milchglastrübungen sowie beginnende Konsolidierungen.

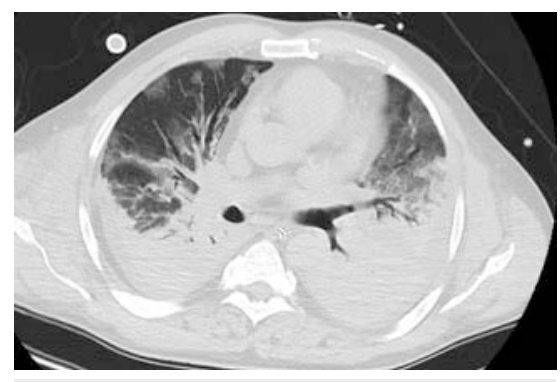

- Abb. 3 Weitere 5 Tage später fanden sich dorsobasal betonte Konsolidierungen, ausgedehnte Milchglastrübungen in den Lungenmittelfeldern sowie relativ gut ventilierte Areale in den ventralen Lungenabschnitten - klinisch und CT-morphologisch lag ein ARDS vor. 


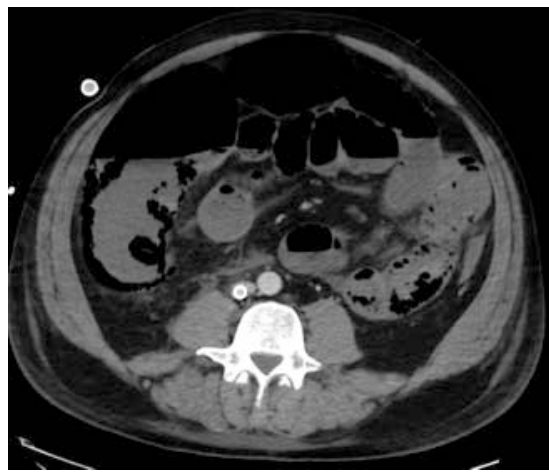

Abb. 4 Ausgedehnte Pneumatosis intenstinalis bei global avitalem Darm. Intraoperativ bestätigte sich dieses Bild.

\section{Diskussion}

Radiologische Untersuchungsverfahren wie die Röntgen-Thorax-Übersichtsaufnahme oder die thorakale CT spielen eine wichtige Rolle, Pneumonien zu identifizieren, und sind fester Bestandteil diagnostischer Algorithmen. Die häufigsten CT-morphologischen Veränderungen einer Influenza-Pneumonie sind bilaterale, periphere, unterlappenbetonte Milchglastrübungen und Konsolidierungen. Häufig besteht ein retikuläres Muster oder ein „Crazy-paving-Muster“, Noduli sind selten. Diese pulmonalen Veränderungen sind unspezifisch und treten auch bei anderen Viruspneumonien auf [Rohani et al. J Intensive Care Med 2016 Jan; 31: 51-60]. Sie überlappen sich deutlich mit denen, die bisher im Rahmen der neuen Lungenerkrankung COVID-19 nachgewiesen wurden. Bei dieser Erkrankung treten ebenfalls vorrangig Milchglastrübungen und Konsolidierungen mit Betonung in den Mittel- und Unterfeldern sowie in der Peripherie der Lungen auf. Meist sind mehrere Lungenlappen betroffen. Pleuraergüsse, Noduli und eine Lymphadenopathie treten in wenigen Fällen auf und sind dann meist Zeichen einer bakteriellen Superinfektion. Aufgrund der prädominanten Milchglastrübungen ist die CT des Thorax der Röntgen-Thorax-Übersichtsaufnahme diagnostisch überlegen. Die häufigsten klinischen Symptome einer Sars-CoV-2-Infektion sind Fieber, Husten, Dyspnoe und Myalgien Symptome, die in ähnlicher Häufigkeit bei Influenza-Patienten auftreten [Kanne et al. Radiology 2020; 27: 200 527].

\section{Schlussfolgerung}

Trotz steigender Vigilanz für Erkrankungen, die durch das neue Sars-CoV-2 ausgelöst werden, sollten saisonale Atemwegserkrankungen wie die durch das Influenzavirus ausgelöste Pneumonie differenzialdiagnostisch bedacht werden. Klinisch und CTmorphologisch zeigen beide Erkrankungen Überschneidungen. Eine Unterscheidung ist mittels PCR möglich, die allerdings im Fall von Sars-CoV-2 eine Sensitivität von nur $60-70 \%$ aufweist. In den Frühstadien von COVID-19 können Veränderungen im CT-Thorax mild ausgeprägt sein oder fehlen. Daher ist eine genaue Risikobewertung (Reiseanamnese, Kontakte zu Erkrankten) von enormer Bedeutung [Kanne et al. Radiology 2020; 27: 200 527]. Fulminante Verläufe sind bei beiden Erkrankungen bekannt. Bis zum jetzigen Zeitpunkt (Stand März 2020) gibt es lediglich gegen die Influenza mit Neuraminidasehemmern wie
Oseltamivir eine kausale Therapie. Erste klinische Studien zur Erprobung einer COVID-19-Therapie beginnen derzeit mit der Patientenrekrutierung [www.clinical trials.gov].

Interessenkonflikt

Die Autorinnen/Autoren geben an, dass kein Interessenkonflikt besteht.

\section{Autorinnen/Autoren}

Sven Wichmann ${ }^{1}$, Vera Spoddeck ${ }^{2}$, Rene Aschenbach ${ }^{1}$

1 Institute for Diagnostic and Interventional Radiology, Jena University Hospital, Jena, Germany

${ }^{2}$ Center for Emergency Medicine, Jena University Hospital, Jena, Germany

Korrespondenzadresse

Dr. Sven Wichmann

Institute for Diagnostic and Interventional

Radiology, Jena University Hospital

Am Klinikum 1

07747 Jena

Germany

Tel.: ++ 49/36 41/9324868

sven.wichmann@med.uni-jena.de

Bibliografie

DOI https://doi.org/10.1055/a-1150-8261 Online-Publikation: 8.4.2020

Fortschr Röntgenstr 2020; 192: 870-871

(c) Georg Thieme Verlag KG, Stuttgart · New York ISSN 1438-9029 